\title{
Traduire la Philanira de Claude Roillet, ou, le laboratoire de la forme poétique théâtrale
}

\author{
JOHN NASSICHUK \\ Western University
}

\begin{abstract}
Claude Roillet, professeur de lettres aux collèges de Bourgogne et de Boncourt, fit paraître en 1556 le recueil de ses cuvres de poète et de dramaturge sous le titre Varia Poemata (Paris: Guillaume Julien). Cette collection contient notamment quatre tragédies latines, intitulées Philanira, Petrus, Aman et Catharina. De ces quatre pièces, seule la première, Philanira, devait faire l'objet d'une traduction française publiée en 1563 aux presses parisiennes de Richard Thomas, puis rééditée, toujours à Paris, chez Nicolas Bonfons en 1577. La présente étude examinera les détails de cette version française quant à (1) l'adaptation en vernaculaire des formes métriques latines variées dont use l'auteur et (2) l'équivalence des formulations lexicales, voire les stratégies d'imitation, dans un texte français qui fait preuve d'une tendance à l'amplification à partir de la composition latine originelle.
\end{abstract}

In 1556, Claude Roillet, a literature professor at the Collège de Bourgogne and Collège de Boncourt, published a collection of his works as a poet and dramatist under the title Varia Poemata (Paris: Guillaume Julien). This collection notably contained four Latin tragedies, which were titled Philanira, Petrus, Aman, and Catharina. Of these four works, only the first, Philanira would become the subject of a French translation published in 1563 by the Parisian press of Richard Thomas, then republished-again in Paris-by Nicolas Bonfons in 1577. This paper will examine the particulars of this French version in terms of (1) the conversion into the vernacular of the various Latin metrical forms that the author uses and (2) the equivalency of lexical expressions, and even the strategies of imitation, in a French text that demonstrates a tendency to amplify the original Latin composition.

Parmi les Varia poemata que Claude Roillet, professeur de grammaire
et de lettres au collège de Bourgogne et au collège de Boncourt fait paraître à Paris en 1556 aux presses de Guillaume Julien ${ }^{1}$, les lecteurs curieux rencontrent, outre de nombreuses épigrammes adressées à des contemporains, trois " dialogues"(Vinearia, Fortunae conjugium, Diana sive Satyri), une " églogue » adressée au cardinal de Lorraine, une épithalame et quatre tragédies latines (Philanira, Petrus, Aman, Catharina) ${ }^{2}$. De celles-ci, seule la

1. Claudii Roillet Belnensis varia poemata (Paris : Guillaume Julien, 1556). Toute référence à l'œuvre latine de Roillet dans la présente étude renverra à cette édition désignée par le titre abrégé Varia poemata. 2. Sur l'œuvre dramatique de Claude Roillet, et en particulier les trois tragédies latines aux thèmes chrétiens, voir notamment R. Lebègue, La tragédie religieuse en France: les débuts (1514-1573) (Paris : 
première à paraître dans l'ordre linéaire du recueil, la Philanira, connut une traduction en langue française et, partant, une postérité éditoriale, comme en témoignent les éditions vernaculaires publiées en 1563 et en 1577, chez Thomas Richard $^{3}$ et Nicolas Bonfons ${ }^{4}$. Dans l'excellente édition qu'elle a procurée de la traduction française de Philanira au sein de la collection « Théâtre français de la Renaissance $»^{5}$, D. Mauri signale justement que la pièce de Roillet fut inspirée quant à son intrigue d'une source narrative italienne, s'agissant vraisemblablement d'une nouvelle de Matteo Bandello ${ }^{6}$. L'action de la pièce porte aussi des ressemblances frappantes avec des récits de Giambattista Giraldi Cinthio et d'Henri Estienne, tous les deux plus tardifs que la pièce de Roillet quant à leur date de publication. D. Mauri suggère également que Roillet luimême procura la traduction française de sa pièce latine originelle ${ }^{7}$, affirmation d'apparence tout-à-fait raisonnable mais qu'aucun document de l'époque ne permet d'affirmer avec certitude, étant donné que Roillet n'attache aucune remarque ni aucune préface aux deux éditions vernaculaires ${ }^{8}$. Nous proposons dans la présente étude une analyse qui cerne quelques spécificités de la version française dans son rapport souvent problématique, parfois révélateur, au texte latin qui la précède. Celle-ci constitue en effet, l'analyse doit le montrer, un véritable laboratoire d'expérimentation linguistique à une époque où le théâtre

Honoré Champion, 1929), p. 259-288. Pour des analyses ponctuelles et plus détaillées des pièces individuelles, je me permets de renvoyer également aux articles suivants : J. Nassichuk « Courage apostolique et crise d'exemplarité : le Petrus (1556) de Claude Roillet », numéro spécial dirigé par A. Graham et L. Frappier, Tangence 104 (2014) : 79-105 ; J. Nassichuk, «A Woman Saint in the Parisian Colleges: Claude Roillet's Catharinae Tragoedia », Renaissance Studies (Oxford) 30.4 (2016) : 562-583.

3. Philanire, tragédie française du Latin de Claude Roillet (Paris: Thomas Richard, 1563).

4. Tragédie françoise de Philanire, femme d'Hypolite (Paris : N. Bonfons, 1577).

5. «Philanire ", texte édité et présenté par Daniela Mauri, dans : La tragédie à l’époque d’Henri II et de Charles IX, première série, vol. 2 (Florence, Paris : L.S. Olschki, Presses universitaires de France, 1989), p. 125-214. Toute référence ultérieure à la version française de Philanira renverra à cette édition.

6. D. Mauri, «Philanire », in La tragédie à lépoque d'Henri II..., p. 132 : «Du moins en partie, le thème de cette tragédie peut être retrouvé dans plusieurs contes italiens du $\mathrm{XV}^{\mathrm{e}}$ siècle. On peut rappeler un conte de Matteo Bandello (II, 15), le seul auteur d'une certaine importance qui ait publié avant Roillet un texte qui peut être rapproché de la tragédie qui nous concerne. »

7. D. Mauri, «Philanire», p. 128.

8. Voir l'article de N. Hugot, sur la Philanira de Roillet, à paraître prochainement in Le Théâtre néo-latin en France (1500-1630). Actes du colloque international de l'université de Bourgogne 22-24 octobre 2015, dir. M. Ferrand, P. Galand et S. Laigneau (Genève : Droz, 2017). 
d'inspiration antique élabore les normes d'usage, au niveau tant du lexique que de la forme, que le siècle suivant cherchera à fixer d'une manière durable.

Libre de toute prétention à l'exhaustivité dans le présent format, notre examen de la pièce de Roillet privilégiera la matière poétique et dramatique du premier acte, qu'il convient de situer ici dans le contexte de l'ensemble. À l'ouverture de la pièce, l'héroïne prononce un monologue plaintif dans lequel elle déplore l'absence de son mari - innocent selon elle - actuellement retenu en prison par le duc piémontais nommé Sévère. Ses deux suivantes surviennent et essaient de l'encourager. Suivant leurs conseils, elle se dirige vers le palais du duc, où elle se met à genoux devant lui ; or, Sévère se montre peu apte à bien recevoir sa prière et lui apprend que son mari doit mourir pour les " crimes » qu'il aurait commis, à moins que l'épouse ne consente à passer une nuit avec son persécuteur, soit le duc lui-même. Au deuxième acte, on assiste à la délibération difficile de Philanire qui accepte finalement le marché du duc Sévère malgré les mauvais pressentiments qu'il lui inspire. Lacte se clôture sur un chant choral qui loue les vertus du lien conjugal et dénonce les violences de Cupidon. Au troisième acte, après avoir passé la nuit avec le duc, Philanire apprend qu'elle n'a été que la victime d'un marché déloyal de la part de celuici, lorsqu'il jette devant elle le corps de son mari décapité. L'acte s'achève sur un nouveau monologue de Philanire et le chant du chœur qui lui offre des consolations. Ensuite, dans le quatrième acte, le vice-roi de France intervient à titre d'ambassadeur et porte-parole de la justice transalpine. Il accuse Sévère de meurtre et ne craint pas d'exiger qu'il répare son injustice en épousant la veuve, Philanire, de sa victime, Hippolyte ! Enfin, au cinquième acte, nouvelle bizarrerie : un messager arrive et raconte au chœur que le vice-roi a décidé de faire tuer Sévère pour lui infliger une punition dont la dureté soit égale à celle de son crime. À la fin de la pièce, Philanire prononce une dernière lamentation sur l'injustice qui la prive de ses deux maris...

Dans l'intérêt de dégager les spécificités de la traduction française à l'endroit de son expression versifiée, nous examinerons la matière poétique, principalement du premier acte, sous trois aspects particuliers: (1) le monologue de l'héroïne qui ouvre la pièce dans un discours de 132 vers heptasyllabiques ; (2) le dialogue hétérométrique (heptasyllabe, octosyllabe, décasyllabe) entre Philanire et ses deux servantes, qui constitue la première "scène " de la tragédie après le monologue expositoire ; (3) le chant choral hétérostrophique qui clôture le premier acte. La comparaison de ces trois moments textuels avec 
la version latine fondatrice montrera à quel point les difficultés formelles et les enjeux sémantiques de la traduction vernaculaire entraînent une véritable reconstitution de la pièce, non seulement au niveau de l'expression poétique, de la facture des vers, mais aussi (et surtout) à celui de la construction proprement dramatique de l'intrigue, dans la mesure où celle-ci est directement concernée par la représentation des " caractères » et de la "pensée », au sens aristotélicien de ces termes?.

Aux rares occasions où elle s'est intéressée à l'œuvre dramatique de Claude Roillet, la critique n'a pas hésité à souligner une forte affinité de l'auteur pour les pièces latines attribuées à Sénèque, y compris justement dans ses aspects techniques. Nous avons pu montrer ailleurs qu'à plusieurs endroits de son œuvre tragique, notamment dans les chants choraux, Roillet déploie une pratique de l'imitation textuelle qui procède de la réécriture de certains passages clés, empruntés directement aux textes du modèle ancien ${ }^{10}$. Il s'ensuit naturellement que le poète dramatique qui reste aussi proche de ses textessources finit par en assimiler plusieurs tournures précises dont il s'efforce de préserver les formes d'expression, tant au niveau de la syntaxe qu'à celui de la métrique. La portée de ce constat philologique nous paraît considérable, puisque les formes constituent chez Roillet de véritables modalités de la construction psychologique des personnages dont elles permettent de saisir les changements de voix et de tonalité. On constate une tendance analogue, affaiblie toutefois par les nombreux enjeux de la traduction, dans la version française de Philanira, tendance largement déterminée par le souci de varier le rythme et la structure des vers. Le traducteur cherche en effet à suivre de près les mouvements du texte originel, s'efforçant de se rapprocher du latin dans le sens et dans le style. Une telle entreprise se trouve naturellement compliquée par la quête d'une syntaxe et d'une forme rythmique en français, dont l'ensemble soit commensurable aux structures des vers latins ${ }^{11}$. Remarquons aussi que le travail de la forme n'est

9. Aristote, Poétique, éd. et trad. J. Hardy (Paris : Les Belles Lettres), p. 38, 1450a.

10. J. Nassichuk, "Echos du chœur des Troades dans l'œuvre dramatique latine de Claude Roillet (1556) ", in Sénèque dans l'Europe des XVI et XVII siècles, dir. M.-N. Fouligny et M. Roig-Miranda, Europe XVI-XVII no. 23 (Nancy : Presses universitaires de Nancy, 2016) : 103-120.

11. Dans cette préoccupation notre travail se rapproche de celui d'E. Borza qui réfléchit sur la problématique analogue de la traduction vernaculaire de la tragédie grecque. Voir son article " La traduction de tragédies grecques : Alessandro Pazzi de’ Medici et les problèmes liés à la métrique », in 
pas toujours facilité par la recherche lexicale grâce à laquelle le poète de langue vernaculaire s'efforce de maintenir le sens du texte qu'il traduit.

\section{Le monologue mono-métrique}

Une telle recherche de l'équivalence formelle constitue ainsi, comme l'a bien montré K. Meerhoff, le lieu d'une réelle complexité technique, puisque la tâche du traducteur exige qu'il accomplisse simultanément plusieurs opérations distinctes et même différentes de nature ${ }^{12}$. Ces enjeux se révèlent aussi avec éclat, dans le domaine de la syntaxe, dès le premier acte de la pièce. Philanire $\mathrm{y}$ apparaît seule sur la scène où elle prononce un long monologue, remplissant ainsi la fonction expositoire qui appartient souvent au personnage central, dont la méditation intime révèle de façon directe les enjeux de l'action. Elle ouvre son discours d'une manière qui la rapproche de maintes héroïnes tragiques anciennes, en se référant longuement à la rotation stellaire et à la succession de la nuit et du jour, images cosmiques qui soulignent l'immensité de sa solitude. L'héroïne se livre à une méditation poétique sur les mystères de la nuit et les frayeurs qu'elle inspire, qui n'est pas sans ressembler au lieu commun maintes fois visité des poètes de la Renaissance de Pontano et Ronsard à Du Bartas et le Tasse $^{13}$. Il s'agit aussi d'un développement lyrique qui éloigne d'emblée le poète et dramaturge de sa source romanesque.

Dans un rêve nocturne, elle voit la triste figure décolorée de son mari absent et emprisonné qui lui annonce sa mort tout en l'implorant de le faire enterrer dignement. Le motif des temps du jour et de la nuit encadre l'ensemble de ce discours qui dans la version latine compte soixante-deux vers anapestiques. Au début de cette longue méditation sombre, qui livre aux spectateurs un aperçu de la situation psychologique de l'héroïne, Roillet situe un développement en deux parties elles-mêmes tripartites, où Philanire déclare que quelle que soit la disposition des cieux, la nuit ne lui cache jamais le songe terrible qui l'obsède de façon quotidienne. La protase de cette déclaration prend la forme d'un tricolon à travers lequel l'héroïne évoque les divers placements des corps célestes :

The Early Modern Cultures of Neo-Latin Drama, éd. P. Ford et A. Taylor (Louvain : Leuven University Press, 2013), p. 63-84.

12. K. Meerhoff, Rhétorique et poétique au XVI siècle en France : Du Bellay, Ramus et les autres (Leiden : E.J. Brill, 1986), p. 25 ss.

13. D. Ménager, La Renaissance et la nuit (Genève : Droz, 2005), p. 26 ss. 
Seu vaga fuscis nox tegit alis

Magna umbrosae bracchia terrae :

Seu purpureis Aurora micans

Bigis, croceum lumen revehit :

Seu flammivomis invectus equis

Pleno Phoebus lucet in orbe... ${ }^{14}$

Ces vers, on le voit, s'organisent autour de la récurrence anaphorique de la conjonction «seu » qui introduit successivement plusieurs situations possibles, chacune desquelles devient la matière de deux anapestes. Le poète maintient ainsi dans l'apodose une certaine symétrie des proportions, puisqu'un nouveau tricolon anaphorique, construit sur la répétition de l'adverbe de négation " non ", décrit l'impuissance de l'obscurité nocturne à voiler ce rêve dont la régularité obsédante se trouve ici reflétée dans les cadences de l’anaphore:

Non nox tenebris curas operit,

Non rore lavat aurora suo,

Non igne fugat Titan aureo. ${ }^{15}$

La figure de pensée construite en deux temps qui ouvre la méditation sombre de Philanire, se conclut ici sur un mouvement d'emphase produit par l'accélération relative des récurrences anaphoriques, lesquelles apparaissent évidemment dans chaque vers de la séquence. Un tel changement de rythme souligne la certitude, voire le sentiment d'urgence, de celle qui parle lorsqu'elle affirme que la vision nocturne revient sans faille toutes les nuits. Dans le texte latin, l'intensification des récurrences et la brièveté relative de l'apodose permettent d'induire aussi en partie la brutalité inquiétante de l'expérience continuellement revécue par la jeune femme.

Ce genre d'apparition outre-tombe constitue un lieu commun fréquemment rencontré dans le théâtre ancien ${ }^{16}$. Les dramaturges de la

14. Varia poemata, f. $1 \mathrm{r}^{\circ}$.

15. Varia poemata, f. $1 \mathrm{r}^{\circ}$.

16. Ainsi Sénèque, Agamemnon (ombre de Thyeste) et Thyestes (ombre de Tantale) ; Euripide, Hécube (ombre de Polydore), etc. Voir O. Millet, « Lombre dans la tragédie française (1550-1640), ou l'Enfer sur la terre ", in Tourments, doutes et ruptures dans l'Europe des XVI et XVII siècles, Actes du colloque de Nancy (Paris : Champion, 1995), p. 163-177. 
Renaissance n'hésitent pas à l'exploiter, aussi la manifestation du mari défunt dans les rêves nocturnes de son épouse porte-t-elle une certaine ressemblance avec les évènements analogues racontés par Cléopâtre dans la première tragédie d'Etienne Jodelle ${ }^{17}$. Souvent cette expérience onirique devient le domaine privilégié de la révélation intime, puisque le revenant qui se manifeste est un personnage déjà largement présent dans le monde "intérieur » de l'héroïne ${ }^{18}$. Les poètes dramatiques de langue vernaculaire s'inspirent par moments, dans l'invention de ces discours intimes, du lyrisme amoureux fortement à la mode à l'époque surtout de la jeune Pléiade ${ }^{19}$. Malgré la date plus tardive de la version française, on remarque sans peine la même tendance, notamment dans ce premier acte où le traducteur s'efforce de bien mettre en évidence le drame personnel de Philanire. Le labeur de la recherche lexicale conduit naturellement le poète français à amplifier à certains endroits la matière de sa source latine. Lorsqu'il traduit la protase de ce premier développement sur la terreur du rêve nocturne, il accorde quatre vers à chaque bras du premier tricolon, redoublement qui n'excède certes pas les proportions habituelles des traductions de textes poétiques latins. Il oblige néanmoins le traducteur à y insérer quelques ajouts qui favorisent le maintien des schèmes à rimes :

Soit que la nuit vagabonde

Ses ailes noires branlant,

Couvre les grands bras du monde

Le clair soleil reculant,

Soit que la vermeille Aurore,

Jaunissant sur ses chevaux,

Le ciel de son teint colore

Et ramène nos travaux,

17. Etienne Jodelle, Cléopâtre captive, éd. K.M. Hall, (Exeter, U.K. : Exeter University Press, 1979), p. 7-9, vv. 63-168.

18. E. Buron, «La renaissance de la tragédie ou le spectacle de la parole : vue et parole dans les tragédies d'Etienne Jodelle ", in Linscription du regard. Moyen Age, Renaissance, éd. M. Gally et M. Jourde (Fontenay-aux-Roses : ENS Éditions, 1995), p. 127-168. Repris dans Lectures d'Etienne Jodelle. Didon se sacrifiant, éd. E. Buron et O. Halévy, (Rennes : Presses Universitaires de Rennes, 2013), p. 139-169.

19. F. Dobby-Poirson, «Le vocabulaire des émotions tragiques des Cléopâtre aptive d'Etienne Jodelle et Médée de Jean de La Péruse ", in Vocabulaire et création poétique dans les jeunes années de la Pléiade (1547-1555), éd. M.-D. Legrand et K. Cameron (Paris : Honoré Champion, 2013), p. 293-306. 
Soit que des sources marines

Phébus ses coursiers tirant,

Par leurs flambantes narines

Voise ce monde éclairant... ${ }^{20}$

Tout en s'efforçant de suivre de près le texte latin, dont il cherche à reproduire la matière lexicale comme en témoigne la formule "nuit vagabonde " pour vaga...nox, le poète vernaculaire s'astreint à maintenir la longueur identique des trois membres de la protase, ce qui l'amène parfois à créer des vers supplémentaires qui préservent aussi la rime. Le quatrième vers, à titre d'exemple, ne correspond directement à aucun élément lexical dans le premier colon latin, mais existe pour préserver l'équilibre de la version française en complétant la rime embrassée. On observe la même pratique dans le quatrième vers du deuxième membre - «Et ramène nos travaux » - où le poète, afin d'achever la rime et de préserver l'équilibre des membres du tricolon, recourt à un lieu conventionnel de l'éloge de la nuit, selon lequel l'apparition du soleil « ramène » toujours les «travaux » de la journée ${ }^{21}$. Parfois aussi, certains mots latins posent un défi considérable à celui qui cherche à préserver l'essentiel du texte latin. On peut signaler à cet égard l'épithète composée flammivomis, dont l'emploi latin s'inspire possiblement d'un passage de Martianus Capella ${ }^{22}$, et que le traducteur relève avec élégance en y substituant la formule «flambantes narines » qui fait représenter la flamma par le moyen d'une épithète. En somme, ces vers assemblent un propos qui reste proche de la version originale, mais les exigences formelles de la composition poétique française, notamment celle de la rime, conduisent l'auteur à amplifier le texte en y insérant des éléments nouveaux. Dans les séquences où il use de cet expédient, le poète français tend à puiser les images dans un fonds conventionnel qui se greffe assez facilement au discours primitif.

Lorsqu'il cherche à reproduire l'apodose de cette déclaration énergique qui ouvre la longue réflexion de Philanire, le traducteur préserve le régime d'équivalences établi dans la protase. Il n'apporte, à la longueur des membres du

20. « Philanire» éd. D. Mauri, p. 146, v. 1-12.

21. D. Ménager, La Renaissance et la nuit, p. 36-39. Voir aussi du même auteur, « Le Nox erat de Virgile et la poésie de la Renaissance ", in Mélanges André Gendre (Université de Neuchâtel, Droz, 2000), p. 1-13.

22. Martianus Capella, De nuptiis Philologiae et Mercurii, I, 70, éd. J. Willis, Leipzig : Teubner Verlagungsgesellschaft, 1983), p. 22. 
tricolon, aucun changement qui corresponde à l'effet d'intensification que l'on trouve dans le texte latin où chacun de ces membres compte désormais un seul vers. L'effet principal dans la version française est l'affaiblissement de la tonalité d'urgence créée en latin par l'accélération des récurrences anaphoriques. Au lieu d'accélérer les répétitions, les vers de l'apodose en français continuent sur le même rythme que dans la protase :

La nuit toutefois ne cache

De son ténébreux manteau

L'ennui qui me grève et fâche

Et me ronge le cerveau.

L’aurore de sa rosée

Ne peut laver les durs plaints,

Dont mon âme est arrosée

Et mes esprits sont tous pleins.

Le soleil qui tout enflamme

Ne peut hors mon cœur chasser

Ce que l'amoureuse flamme

M'y a voulu pourchasser. ${ }^{23}$

On assiste dans cette séquence à un effet de ralentissement du discours qui est le résultat immédiat d'un choix de traducteur. Au lieu de maintenir les proportions relatives qui caractérisent le texte latin, le poète français construit une séquence dans laquelle la protase et l'apodose procèdent désormais au même rythme. Le schème à rimes, qui constitue l'unité formelle dominante dans le monologue de Philanire, conduit le poète à écrire un discours composé de quatrains à la rime imbriquée. A chacun des vers singuliers, qui occupe à lui seul un membre du tricolon de l'apodose dans le texte latin, l'auteur de la version française substitue un quatrain entier constitué d'une seule phrase.

Un tel changement dans les proportions du texte devient aussi l'occasion d'ajouts lexicaux qui ne manquent pas d'amplifier la matière du discours. Dans le premier des trois quatrains, le poète traduit l'ablatif tenebris par l'emploi 
d'une métaphore - «ténébreux manteau » - absente du latin ${ }^{24}$. L'accusatif au pluriel curas est traduit par un vers tout entier, dans lequel une relative subordonnée - « qui me grève et fâche » - s'ajoute au substantif initial afin de remplir l'heptasyllabe en préservant la rime. À sa suite, le vers ultime de ce quatrain - «Et me ronge le cerveau » - ne correspond à aucun élément lexical dans le vers latin, mais il demeure nécessaire afin de compléter harmonieusement l'unité rimée. Au second quatrain, le substantif " plaints » fournit au verbe «laver » un objet qui n'apparaît pas dans le vers latin, et les deux derniers vers de cette unité ne correspondent à aucun élément lexical apparaissant dans la version originale. Enfin, le dernier quatrain du tricolon en français attribue au verbe "chasser » un objet également absent du vers latin qu'il est censé traduire. L'effet d'amplification dans le texte vernaculaire est ainsi doublement mis en évidence par le fait que le traducteur attribue à chaque verbe un objet séparé, alors que le latin structure l'usage répété de formes verbales autour d'un seul substantif : curas.

Une telle économie de l'expression latine fait accroître de toute évidence le défi qui s'impose au traducteur en soulignant naturellement les différences d'ordre syntaxique entre les deux langues. Il en résulte une prolongation de l'apodose qui, en la ralentissant, la prive aussi del'esprit volontaire quel'anaphore énergique lui donne dans la version latine. Cet émoussement de l'insistance rapide et répétitive amène des conséquences non seulement au niveau de la traduction littérale des vers, mais aussi à celui de l'effet dramatique exercé par ce discours d'ouverture. En conférant à l'anaphore qui structure l'apodose un caractère plus lyrique, voire intime, grâce aux effets de l'amplification, le poète français tend à construire un discours davantage axé sur la réflexion mélancolique du personnage solitaire.

\section{Le dialogue hétérométrique}

La solitude de Philanire ne tient pas uniquement au fait qu'elle apparaît seule sur la scène dans son monologue d'ouverture, loin s'en faut, car la mentalité solitaire est inscrite en quelque sorte déjà dans les cadences rythmiques des paroles latines prononcées par le personnage dès le premier acte, y compris

24. La formule n’est évidemment pas sans évoquer la célèbre expression "fabuleux manteau » déployé par Ronsard au début de l'« Hymne de l'Automne». 
lorsqu'elle se trouve accompagnée de ses servantes. Cette disposition mentale de l'hérö̈ne est bien mise en évidence par le jeu de contrastes que Roillet ne manque pas de faire apparaître dès ce que l'on pourrait appeler la deuxième " scène " du premier acte de la pièce, laquelle réunit en effet les deux « demoiselles » suivantes de Philanire, autour de leur maitresse éplorée. Après le long monologue anapestique prononcé par Philanire à l'ouverture de la pièce, cette deuxième scène, qui ouvre donc la partie dialoguée, est composée principalement de trimètres ïambiques confectionnés à l'imitation de Sénèque chez qui c'est la forme préférée dans les séquences de dialogue. Principalement, car la séquence de trimètres, qui se prolonge jusqu'à l'irruption du chant choral à la fin de cette réplique de 104 vers, est subitement interrompue lors de la deuxième réplique prononcée par Philanire, consistant en une séquence de cinq vers dans lequel le poète revient soudain au rythme anapestique du monologue. L'échange entre les trois personnages souligne avec clarté la distinction entre l'héroïne et ses deux subalternes, mettant bien en évidence la solitude discursive et "métrique ", la cadence singulière, de celle qui réfléchit toujours au destin de son mari prisonnier :

\section{Prima Ancilla.}

Dum vix Olympo Aurora pellit lucida

(trimètres ïambiques)

Sydera, micans Titan coma vix dum aurea

Reddit diem, quaestus misera repetis tuos.

\section{Philanira.}

Quid non repetam, et vincam Halcyones, (bimètres anapestiques)

Vincam et volucres Pandionias,

Gravior quando his fortuna mea est?

Semper meus est mihi vir flendus,

Vir primorum causa dolorum. ${ }^{25}$

Ce changement de rythme ne dure guère, car le même personnage reprendra les cadences solennelles du trimètre dès sa réplique suivante, quatre vers plus loin : 


\section{Secunda Ancilla.}

Nullum tuis si luctibus ponis modum,

(trimètres ïambiques)

Si semper artus intimus rodit dolor,

Si fletus exedit animum, ecquod gaudium

Sperare possis? quod tibi vitae genus?

\section{Philanira.}

Vitae genus suave est mihi breviter mori. ${ }^{26} \quad$ (trimètre ïambique)

La variation soudaine correspond au radoucissement de la voix de Philanire lorsqu'elle souligne la légitimité de ses inquiétudes, voire de sa tristesse prémonitoire, face aux encouragements un peu trop sanguins proférés par ses deux servantes. Comme elle constitue le seul changement de valeur métrique dans toute la scène, cette variation de cadence métrique souligne aussi l'individualité de l'héroïne et l'isolement qui l'afflige même lorsqu'elle se trouve entourée d'amies.

Dans la version française publiée treize ans après la parution de l'original latin, le monologue prononcé par Philanire au début de la pièce contient 132 heptasyllabes en rimes imbriquées, avec une alternance des désinences féminines et masculines. Tout porte donc à croire que le vers heptasyllabique correspond dans ce discours d'ouverture à l'anapeste latin, puisqu'il occupe plus ou moins le statut de vers "lyrique " analogue à celui que Sénèque tragédien déploie fréquemment dans les chants choraux ${ }^{27}$. En revanche, la traduction de la séquence dialoguée située à sa suite devient l'endroit d'une complexité métrique et formelle qui l'éloigne sur plusieurs points, ici encore, de la version latine où une seule réplique de Philanire se démarque de tous les autres propos proférés par les trois personnages. Les premières répliques de la séquence sont débitées en vers octosyllabiques, forme métrique associée par les théoriciens de l'époque, comme aussi l'heptasyllabe, au lyrisme du sonnet et de l'ode. Ainsi, Jacques Peletier du Mans fait observer que ces deux types de vers conviennent à " l'ode sérieuse ", alors que le vers en six syllabes

26. Varia poemata, f. $2 \mathrm{v}^{\circ}$.

27. J.G. Fitch, Seneca's anapaests : metre, colometry, text, and artistry in the anapaests of Seneca's tragedies (Atlanta : American Philological Association, Scholar's Press, 1987). 
est plus convenable à des matières joyeuses et légères ${ }^{28}$. Dans ce contexte néanmoins, l'octosyllabe qui ouvre le dialogue marque toujours une certaine différence par rapport à l'heptasyllabe usité dans le monologue de l'héroïne. Aux interrogations des servantes sur la cause de sa tristesse, celle-ci répond qu'elle pleure tout naturellement sur l'emprisonnement de son mari. Sa brève réplique s'achève sur l'ouverture d'une rime plate féminine que le propos de la première servante viendra compléter :

\section{Philanire.}

En pleurant passe la douleur : (octosyllabes)

Et pourquoi aussi au malheur

De mon mari serai heureuse?

\section{$1^{\text {ère }}$ demoiselle.}

A peine l'aurore joyeuse (octosyllabes)

Chasse les étoiles des cieux,

Titan de ses radiants yeux

Découvrant sa perruque blonde,

A peine éclaire ce bas monde,

Et vous (las) déjà desmachés

Vous plaintifs et vous pleurs cachés. ${ }^{29}$

Lorsque Philanire prononce sa deuxième réplique de la scène, formulée en anapestes dans le texte latin, le traducteur français revient au mètre $\mathrm{du}$ monologue d'ouverture, l'heptasyllabe. La recherche d'une certaine correspondance formelle entre les deux versions de la pièce transparaît jusqu' ici avec netteté. Or, c'est bien à partir de ce moment que les séquences latine et française se développent de deux façons distinctes. Puisque la réplique de la "première demoiselle », prononcée en vers octosyllabiques se termine sur une rime plate achevée, la réponse de Philanire peut se construire librement, en dehors de toute contrainte formelle à l'égard des vers qui la précèdent. Voici donc l'instant où le traducteur choisit d'opérer un changement de mètre et de

28. Jacques Peletier du Mans, Art poétique, II, i, «Des vers français » dans : F. Goyet éd., Traités de poétique et de rhétorique de la Renaissance (Paris : Librairie générale française, 1990), p. 213 : «Ceux de six se mettent commodément ès Odes, principalement, quand ce sont des choses gaies. J'y ai décrit mon Rossignol. De sept et de huit, sont fort fréquents, et capables de l'Ode sérieuse. » 29. « Philanire », éd. D. Mauri, p. 150-151, v. 151-160. 
rime qui correspond au glissement du trimètre à l'anapeste dans cette même partie du texte latin. Les cinq anapestes de la Philanire latine se transforment en quinze heptasyllabes lorsque la réplique analogue est prononcée par l'héroïne vernaculaire :

\section{Philanire.}

Las, pourquoi ne le ferai, (heptasyllabes)

Pourquoi ne répéterai

Le comble de ma tristesse?

Pourquoi mon deuil ne dirai

Et de mes pleurs passerai,

Seuls témoins de ma détresse,

Les plaints halcioniens

Et les Pandioniens,

En plus fâcheuse fortune?

Pleurer il me faut les miens

Et le meilleur de mes biens

Qui m'est ravi par rancune.

Hélas, mon pauvre mari

Que je vois presque pourri

En la prison inhumaine. ${ }^{30}$

Le traducteur choisit, on le voit, d'altérer ici le schème à rimes, afin de souligner la singularité des propos de Philanire triste et solitaire. À la suite d'une rime plate initiale, la plainte de l'épouse inquiète s'élabore à travers une séquence consistant d'un «quatrain » de rimes embrassées (fmmf), une rime plate, un nouveau quatrain de rimes embrassées et une deuxième rime plate. Seul le dernier vers de la réplique, qui ouvre une nouvelle rime, demeure sans réponse interne. Sous la contrainte de cette même rime, la « deuxième demoiselle » offre sa réponse dans le même mètre, préservant ainsi le rythme heptasyllabique comme s'il s'agissait pour elle de soutenir le ton identique afin de lui répondre sur le registre de l'intimité solitaire, qui distingue justement l'héroïne de ses deux suivantes dans le texte latin. Ce partage du registre intime entre les deux 
personnages rapproche la version française des dialogues entre l'héroïne et ses suivantes dans les deux tragédies d'Etienne Jodelle.

Ici Philanire donne une nouvelle réplique en deux heptasyllabes, puis le rythme change encore : la première " demoiselle » intervient en reprenant le fil de la thématique des étoiles célestes qu'elle commençait à développer quelques vers auparavant. Parvenu à ce stade, le traducteur introduit une nouvelle variation en attribuant à la première « demoiselle » une série de huit décasyllabes qui correspondent de près à la réplique de cinq trimètres dans la version latine originale :

\section{$1^{\text {ère }}$ demoiselle.}

Déjà Phébus par deux fois s'est levé (décasyllabes)

Et a son chef en l'océan lavé;

Déjà sa sœur en poursuivant sa trace

A par deux fois dessus son char sa face

D’un tain d'argent élancé dessus nous.

Et toutefois de ce temps n'avez-vous

$\mathrm{Ni}$ jour ni nuit fait à la bouche offrande

Tant peu que soit pour goûter de viande. ${ }^{31}$

Cette valeur métrique, introduite ici pour la première fois dans la pièce, s'impose rapidement, l'espace de soixante-dix vers, à tous les personnages. L'octosyllabe redevient ensuite le véhicule de l'expression collective pendant une cinquantaine de vers, avant le retour final du décasyllabe dans la trentaine de vers qui terminent la longue scène dialoguée. Au lieu de suivre de près la version latine quant aux changements métriques, la traduction française de cet échange entre trois personnages féminins devient le moment d'une variation bien dynamique, caractérisée par plusieurs altérations de vers.

Tout suggère donc qu'en passant de la version latine publiée pour la première fois en 1556, à celle qui parut en langue vernaculaire sept ans plus tard, le rapport fondamental qui subsiste entre le discours des personnages et la forme de leur diction acquiert une mesure de complexité supplémentaire, liée aux multiples enjeux formels de la traduction. Alors que dans le texte latin les schèmes métriques s'associent à certaines tonalités voire à certaines situations 
- c'est le cas des anapestes prononcés par Philanire -, le texte vernaculaire tend en revanche à délayer ces associations fortes qui lient le contenu du propos à la forme poétique latine. Un tel changement met le traducteur devant les défis multiples de l'accommodation inventive, car il altère naturellement l'expression dramatique du caractère et de la situation. Alors que Roillet s'appuie fréquemment sur les formes et le lexique de Sénèque dans ses pièces latines, le traducteur vernaculaire se livre à une véritable recherche expérimentale dans la recomposition française de la tragédie.

Afin de bien illustrer le défi qui s'impose au traducteur français lorsqu'il cherche à rendre les vers latins de la Philanira dans une forme vernaculaire qui soit analogue à celle du texte original, deux considérations techniques et formelles s'imposent de prime abord : (1) l'économie relative du texte latin, inhérente à la qualité naturellement synthétique d'une langue flexionnelle ; (2) les exigences de la rime. Aussi quant à cette deuxième considération, celle de la rime, la réplique que Philanire prononce en anapestes dans la séquence dialoguée illustre parfaitement le cas. Si le personnage de langue latine se distingue par le rythme proprement thrénodique qui souligne son isolement sur la scène au milieu même des autres personnages, son homologue vernaculaire entraîne son interlocutrice, la "deuxième servante ", par les cadences heptasyllabiques de ses propos, à lui répondre sur le même rythme. Dans cette séquence composée en rimes plates, Philanire termine sa réplique sur la première moitié d'un couplet : « Hélas, s'écrie-t-elle, mon pauvre mari / Que je vois presque pourri / En la prison inhumaine». La rime est complétée ensuite par la deuxième servante au début de sa réplique : "Soyez vers vous plus humaine ». Outre la pression évidente de la rime, la matière lexicale de la séquence suggère de croire que le traducteur français nourrit ses vers d'une langue empruntée aux poètes contemporains. On peut citer à cet égard la rime masculine qui apparaît chez Ronsard en 1556, dans le sonnet 49 de la Continuation des Amours ${ }^{32}$. Mais il convient surtout de souligner la proximité dramatique de cet échange rimé entre maîtresse et servante, avec une séquence qui apparait sous la plume d'Etienne Jodelle dans sa Cléopâtre captive portée à la scène devant l'auditoire du Collège de Boncourt en 1553, où Charmium,

32. vv. 9-10 : «Es-tu quelque Busire, ou Cacus inhumain, / Pour te saouler ainsi du pauvre sang humain?» 
l'une des deux suivantes de la reine Cléopâtre, reproche à sa maîtresse la dureté implacable dont elle fait preuve à son propre égard ${ }^{33}$.

Des exemples comme celui-ci abondent dans la Philanire, et le texte français mériterait à ce titre des études philologiques, comparatives, bien plus détaillées que les quelques lignes critiques dont elle a fait l'objet jusqu'à présent. La primauté formelle de la rime constitue bien, on le voit, à la fois une manière d'approximation propre à augmenter la force rythmique d'une expression vernaculaire largement dépourvue des valeurs de longueur syllabiques qui demeurent à la base de la versification latine, et une véritable contrainte qui conduit souvent le poète à amplifier son texte. Or, malgré ce défi redoutable inhérent à la traduction d'une langue à l'autre, le traducteur découvre par moments des astuces qui lui permettent de préserver une mesure d'équivalence entre les deux versions. Il convient de citer comme preuve les vers 183-184 dans la traduction française, où l'auteur français rend la réplique de Philanire, laquelle tient en un seul trimètre iambique, par une formulation, presque également brève, en deux heptasyllabes rimés : « Ma joie et ma douce vie / C'est être de mort ravie ». Malgré le redoublement des vers, motivée en grande partie par les exigences de la rime, le compte des syllabes permet de constater que les deux versions sont de longueurs presque identiques.

Plus fréquemment, toutefois, la densité synthétique des valeurs latines s'avère impossible à reproduire, si bien que notre traducteur se contente d'une réécriture amplificatrice apte à reconstituer le sens de l'original. Il sera maintenant utile de contempler, exemple éclatant de cette tendance massive, la manière dont le traducteur s'efforce de traduire le vers 248 de la version latine. Il s'agit d'un nouveau trimètre ïambique divisé en trois parties, car le jeu rapide des voix morcèle le vers, l'écartelant entre autant de répliques! C'est le moment, vers la fin du premier acte, où la pauvre Philanire apprend enfin le sort de son mari et commence à entrevoir avec horreur une part de la cruauté cynique qui motive le duc:

\section{Severus.}

Quid ? tu illius ne es misera conjux

Quem crimen imo deprimi vult carcere?

33. Etienne Jodelle, Cléopâtre captive, éd. K.M. Hall, p. 9, v. 169-170 : (Cléopâtre) « Que gaignez-vous, hélas, en la parole vaine?» (Eras) «Que gaignez-vous, hélas ! de vous estre inhumaine?» 
Cui jura plebis, cui bona eversa et decus?

Quem iam moratur?

Philanire

Ah quid ? Ah!

Severus. Crux vel rota.

Philanire.

Crux vel rota? O dii, siccine juves vivere

Qui me in viro cogis mori ? ${ }^{34}$

Sévère termine ici une première réplique au milieu du troisième iambe, le verbe " mourir » se référant à Hippolyte (Quem iam moratur...). L'emploi du subjonctif jussif qui ponctue la phrase à l'intérieur du vers, suscite l'interjection terrifiée et presque suffoquée de l'héroïne (Ah ! quid, ah !) et permet à Sévère d'achever le trimètre en éclaircissant cruellement le sens de ses propos : Crux vel rota. Dans la version française, l'auteur rallonge cette réplique brutale en en faisant tout un couplet rimé. Aussi déplace-t-il l'emploi du verbe " mourir » jusqu'après la réplique inquiète de Philanire, sans doute afin de préserver à l'échange des deux personnages un niveau de tension dramatique semblable à celui que l'on rencontre dans le texte latin :

\section{Sévère.}

Es-tu donc la femme à celui

Que tiens en prison, et pour lui

Veux prier, qui pour son offense

N'attend que digne récompense?

Pour le peuple contre la loi

Pillé sans l'entente du Roi

Ja de ce jour n'atend que l'heure

Que...

Philanire. Quoi, hélas, quoi ?

Sévère.

Qu'il ne meure,

Ou au gibet étant pendu,

Ou dessous la roue étendu. 


\section{Philanire.}

Sur roüe, hélas, ou gibet ? O puissance

De tous les cieux, est-ce donc l'espérance

Que j'ai en toi de ma vie recouvrer,

Qui veux ainsi à la mort me livrer ? $^{35}$

Lorsqu'il traduit l'annonce terrible que Sévère n'hésite pas à prononcer devant la jeune femme, le poète français use toujours des procédés de l'amplification afin de bien transmettre le sens du texte original. Mais une réponse brutale comme celle de Sévère - Crux vel rota -, dont l'effet réside dans la brièveté choquante de la formulation, pose au traducteur un défi d'ordre dramatique que les reconstructions amplifiées ne sauraient relever d'une manière adéquate. Dans la mesure où la portée dramatique des vers latins repose sur des jeux de longueurs relatives et proportionnelles, notamment lorsqu'il s'agit de révélations formulées d'une manière succincte, la simple traduction du sens perd souvent l'effet du texte latin. Afin de préserver l'effet dramatique de la révélation soudaine si dévastatrice pour l'héroïne, l'auteur de la version française introduit dans la réplique de Sévère un subjonctif — «Qu'il ne meure... - - dont la brutalité rapide vise à produire un effet semblable à celui de la formule latine qui, dans le texte français, occupe désormais l'espace d'un couplet entier.

Autre noyau essentiel, avec le monologue, de l'invention dramatique, la forme dialoguée soumet le traducteur à des exigences qui dépassent souvent de bien loin celles, déjà considérables, de la restitution du sens original. La dynamique des échanges entre les personnages qui apparaissent ensemble sur la scène, conduit en effet l'auteur de la Philanire à changer fréquemment de schèmes de rimes et de mètres au sein d'un même dialogue. Il en résulte un produit bien différent du texte latin où la régularité métrique du débit constitue un fondement suffisamment fort pour que chaque divergence soit chargée de sens dramatique. La tendance à l'amplification, maintes fois attestée dans la version française de Philanira, modifie le ton et l'énergie des propos et, partant, l'importance relative de certaines répliques. Or, la lecture attentive des chants choraux montre aussi qu'elle fait preuve par moments d'une vertu glossatrice susceptible d'éclaircir les obscurités - ou de lever les ambiguïtés - des vers latins. 


\section{Le chant choral hétéro-strophique}

Dans le chant choral qui clôture le premier acte, le poète latin porte un regard sur les origines de la convention matrimoniale. Il profère l'éloge de cette union stabilisatrice qui privilégie les effets sains de la fidélité et de l'affection réciproque, alléguant de nombreux exemples de l'Antiquité païenne qu'il puise dans les ressources mémorielles tant de la mythologie (Vénus, Junon, Hercule) que de l'histoire civile et militaire (Pompée, Gracque). La version latine originale compte quelques soixante-trois vers répartis en douze strophes de longueurs inégales. On y découvre en effet trois unités de huit vers, une autre de sept vers et huit de quatre vers. Malgré cette diversité apparente, le chant du chœur se construit autour d'une forme régulière, le fondement métrique de l'ensemble étant bien représenté par ces huit quatrains, tous constitués de la strophe lyrique asclépiade $\mathrm{A}$, laquelle est formée de trois vers asclépiades à douze syllabes et d'un glyconique à huit syllabes. Quant aux strophes de huit et de sept vers, elles aussi se construisent de six ou de sept asclépiades suivis d'un glyconique. Une forme poétique récurrente, fréquemment attestée dans les Odes d'Horace et dans les chœurs tragiques de Sénèque, constitue ainsi la base inventive du chant choral qui marque la fin du premier acte de la Philanira.

Les deux premières strophes du chant choral évoquent les tenants symboliques de l'évènement matrimonial et des éléments traditionnels associés à la cérémonie festive des anciens. Roillet y déploie un lexique hérité des chants de Catulle, de Plaute, de Claudien :

Qui primus veterum concubitus vagos

Repressit, statuens conjugibus modum, Sacri jura thori, vincla jugalia

Nocturnasque faces, mellaque dulcia

Puro expressa favo, quae biberet, suo

Uxor juncta viro : qui statuit, caput

Ut supra hasta micans, vel gladius for

Quanto judicio fuit?

Hinc Caius celebris, voxque Talassii

Inculcata frequens, et resonans hymen, Hinc auspex sacer, et pronuba nuptiis 
Primis sacra fuit : nupta nova hinc caput

Obduxit tenui candida linteo :

Hinc sparsae pueris dum trepidant nuces,

Hinc far, hinc Veneris cingula Cypriae

Quae vir soluere debeat. ${ }^{36}$

Le poète conclut ainsi l'acte initial de la tragédie sur un nouveau chant du temps de la nuit, qui rejoint de façon ironique la plainte des terreurs nocturnes avec laquelle l'héroïne elle-même ouvre la pièce. Plusieurs éléments lexicaux dans ces vers, tels «nova nupta $»^{37}$, "Talassii $»^{38}$ et la référence aux noix associés aux chants fescennins, témoignent d'une forte influence catullienne. À la fin de la deuxième strophe, il renvoie notamment à la « ceinture » matrimoniale portée par la jeune fille à marier lors de ses noces, la même qu'évoque fameusement Catulle dans son poème LXVII, lorsque la porte d'une maison familiale livre au public les secrets intimes de ses habitants ${ }^{39}$.

Quant à la version française de ce chant choral, elle comporte quatre-vingtdix-neuf vers de longueurs variées, organisés en dix strophes hétérométriques, dans lesquels le traducteur s'efforce de suivre de près le texte latin. De ces dix strophes métriquement identiques, les neuf premières sont des dizains, chacun desquels peut se décomposer en deux parties principales : (1) un quatrain construit de deux décasyllabes rimés qui embrassent un couplet d'octosyllabes; (2) six hexasyllabes consécutifs en rimes plates. Une telle configuration métrique interne tend à souligner le caractère musical de ces strophes, les démarquant ainsi des structures plus régulières, moins variées, des séquences dialoguées. Elle correspond ainsi, dans un rapport de valeurs parallèles, à celle de la strophe lyrique asclépiade A pour la version latine, puisque la ponctuation des dizains par une séquence d'hexasyllabes livre un jeu de proportions analogue à celui que donne l'emploi du glyconique en huit syllabes à la suite des

36. Varia poemata, f. $6 \mathrm{v}^{\circ}$.

37. Catulle, Poésies, texte établi et traduit par G. Lafaye (Paris : Les Belles Lettres, 1958), p. 78 : Cat. LXI, 96-100 : «Prodeas, nova nupta, si / Iam videtur, et audias / Nostra verba. Vide ut faces / Aureas quatiunt comas ; / Prodeas, nova nupta. »

38. Cat. LXI, 131-135 : «Da nuces pueris, iners / Concubine ; satis diu / Lusisti nucibus ; lubet / Iam servire Talasio. / concubine, nuces da. »

39. Cat. LXVII, 20-28 : «Non illam vir prior attigerit,/ ... / Et quaerendus is unde foret nervosius illud / Quod posset zonam solvere virgineam.» 
asclépiades. Or, le choix de cette forme élégamment ciselée impose ici encore un défi considérable au traducteur, en particulier lorsqu'il s'agit pour celui-ci de restituer la matière des strophes latines rallongées qui contiennent sept vers longs avant le glyconique :

O que celui était bien entendu

Qui, réprimant la liberté

Des vagues lits, a arrêté

Un sacré lit, un lien prétendu ;

De nuit torche éclairante

Et la liqueur coulante

Du doux miel retiré

Que l'un et l'autre boit,

Et le glaive reçoit

Dessus le chef tiré.

De la mort de Thalasse on a pris,

Du prêtre saint, et de Junon,

Et du sacré Hymène le nom,

A été mis en la bouche et en pris,

Quand d'une blanche toile

L'épouse son chef voile,

Le mari aux abois

Desçaint pour premier bien

De Vénus le lien

Et épanche des nois. ${ }^{40}$

Dans ce rapport strict et minimaliste du latin au français, qui exige presque la traduction mot à mot du texte original, le poète vernaculaire peine par moments à maintenir le niveau de clarté qui distingue de façon remarquable la version latine. Ainsi, le mode interrogatif de la première strophe, qui souligne la distance réflexive du chœur prêt à remonter dans le temps afin de bien mettre en perspective la situation de l'héroïne, disparait entièrement dans la traduction française. Afin de bien respecter les contraintes formelles de la brièveté, le 
poète français néglige aussi de restituer le sens du génitif veterum, choix qui confirme ce changement de ton et de perspective en omettant le renvoi général à la tradition antique. Disparaissent aussi, dans la deuxième strophe en langue vernaculaire, les résonances du chant matrimonial, lesquels se manifestent avec éclat dans les choix lexicaux du poète latin qui déploie savamment les échos catulliens. La formule Veneris cingula Cypriae / Quae vir soluere debeat, est désormais traduite par une construction plus diffuse, répartie sur trois vers : «Le mari... / Desçaint.../ De Vénus le lien ». Il en résulte une version qui demeure sémantiquement fidèle à l'expression originale, sans reproduire pour autant les effets poétiques de l'écho catullien induit par l'usage du verbe latin solvere.

Lorsqu'il s'aventure, en revanche, à traduire les strophes non rallongées, c'est-à-dire qui contiennent uniquement trois asclépiades et un glyconique, le traducteur use toujours du dizain hétérométrique. Ce maintien de la forme lui permet aussi de livrer, en traduisant des unités abrégées, une version qui préserve davantage le sens et la matière lexicale du texte latin. Pour certaines de ces strophes, la traduction fournit une telle moisson de détails et de précisions que le texte vernaculaire en vient par moments à ressembler à une véritable glose explicative, destinée à faciliter au lecteur la compréhension de l'original ${ }^{41}$. Une lecture de la première de ces strophes plus courtes, la cinquième strophe du chant choral, suffit à le montrer. Dans ces quatre vers, le chœur se félicite des conséquences heureuses de la véritable « institution » civile qu'est devenu le mariage. L'un des principaux fruits de la coutume matrimoniale, affirme-t-il, réside dans l'harmonie sociale qu'elle nourrit. C'est le résultat d'une rencontre charnelle et spirituelle de deux personnes qui proviennent de familles différentes. Ensuite, dans la strophe asclépiade conventionnelle à quatre vers, le chœur explique, par le moyen d'une élégante brièveté, que lorsque l'esprit et le sang se mêlent amoureusement à travers les ébats d'un couple marié, le lien formé émerge tellement puissant que même Hercule ne saurait le défaire :

41. Il s'agit peut-être d'un cas qui reflète la valeur éclairante de la langue vernaculaire, vue par les humanistes français à l’époque de la Pléiade, lorsqu'elle est appliquée à l'explication des textes de langue latine. Voir à ce sujet A. Vintenon, « La traduction comme vitrine du lexique français : la "Fantaisie" dans l'Art poétique d'Horace traduit par Jacques Peletier du Mans ", in Vocabulaire et création poétique..., p. 28 : «La traduction d'un texte latin en français semble constituer un bon terrain d'expérimentation pour montrer que, par la diversité de ses racines linguistiques, le français peut parfois présenter des richesses et une précision que n’offrait pas la version originale. » 
Dum se mixtus agit sanguine spiritus,

Alternasque subit seminibus vices,

Is vincitur amor, solvere quem sua

Dextra non queat Hercules. ${ }^{42}$

Tel est donc le message de ces vers choraux, auxquels l'élégante simplicité de la formulation confère des allures de proverbe. Seule en effet la présence de l'infinitif solvere, qui recèle toujours un souvenir de Catulle, suggère quelque renvoi délibéré à la deuxième strophe du chant choral, dans laquelle la voix collective évoque l'image de la « ceinture de Vénus cyprienne », symbole ancien de l'amour matrimonial.

Ce rapport subtil entre la puissance d'un lien amoureux qu'Hercule luimême ne saurait rompre, et la référence à la ceinture de Vénus dans la deuxième strophe du chant choral, transparaît avec un éclat renforcé dans la traduction française de la strophe asclépiade. Le poète use en effet des modalités de l'amplification pour souligner la continuité thématique qui subsiste entre les deux évocations du « lien » amoureux :

Ce tempendant que l'esprit assembler

Peut par le sang tant seulement

Les deux corps en un tellement,

On ne pourrait l'amour désassembler,

Car il y est de sorte

Que même la main forte

D'Hercule ne pourrait

Dénouer le lien

Du nœud fait Ciprien

Quand il y tâcherait. ${ }^{43}$

L'explication négative qui apparaît au quatrième vers de cette strophe constitue un ajout descriptif qui ne correspond directement à aucun élément de la version latine, dans laquelle seule la particule " ne " dans l'adonique représente une négation. Dansle textelatin en effet, la formulation verbale négative «solvere.../...

42. Varia poemata, $7 \mathrm{r}^{\circ}$.

43. « Philanire », éd. D. Mauri, p. 163, v. 581-590. 
non queat » prend comme objet le relatif « quem » désignant l'amour (amor) du couple marié. Le traducteur restitue à la séquence la métaphore du "lien » déjà introduite dans la deuxième strophe et à laquelle le poète latin fait seulement une référence oblique. En outre, l'épithète toponymique "Ciprien » apparaît ici pour la première fois, le poète souhaitant éviter la répétition du nom propre (Vénus) déjà usité auparavant pour éclaircir le sens du génitif « Cypriae ». Dans ce cas donc, le dizain hétérométrique traduit l'asclépiade de façon à souligner clairement, grâce à l'ajout de précisions lexicales et syntaxiques, la continuité thématique liant cette strophe à celles qui ouvrent le chant du chœur à l'éloge de l'amour conjugal.

Pour conclure, il convient de remarquer que la variété de formes métriques, strophiques et syntaxiques, déployées par le traducteur de la Philanira de Claude Roillet, reflète au moins partiellement la virtuosité technique de l'auteur latin, professeur de grammaire et de lettres aux collèges parisiens pendant les jeunes années de la Pléiade. Ce même traducteur reproduit aussi la tendance expérimentale des premiers dramaturges d'orientation " classique », dont les productions datent de la même période que les Varia poemata. L'analyse du monologue d'ouverture élaboré dans un seul mètre, de l'échange dialogué hétérométrique et du chant choral hétérostrophique, montre certes un traducteur constamment aux prises avec les difficultés inhérentes à sa tâche : trouver un terrain commun entre deux langues, l'une ancienne et l'autre " moderne ", et séparées par leurs régimes syntaxiques si différents l'un de l'autre. Toutefois, elle pointe aussi, à la fois, l'urgence et la fécondité proprement dramatiques de ce labeur d'expérimentation formelle et lexicale qui apparente l'initiative des traducteurs de pièces de théâtre à celle, plus célèbre et mieux étudiée jusqu'à présent, des poètes vernaculaires dont les œuvres composent «le laboratoire horatien des années $1550 »^{44}$. Le terrain d'investigation qui en émerge est bien celui du rapport entre le texte et l'action qu'il nourrit et qu'il provoque, entre l'invention linguistique et la pragmatique du jeu qui constitue désormais, à partir des années 1550 justement, l'un des principaux chantiers des poètes de langue française.

44. N. Dauvois, "Vers courts et strophes lyriques. Le laboratoire horatien des années 1550 ", in L’expérience du vers en France à la Renaissance, éd. J.-C. Monferran (Paris : Presses de l'université ParisSorbonne, 2013), p. 105-123. 
